Biological sciences / Neuroscience / Cognitive neuroscience [URI /631/378/2649]

Physical sciences / Engineering / Biomedical engineering [URI /639/166/985]

Scientific community and society / Business and industry / Technology [URI /706/703/559]

\title{
Neurocognitive considerations to the embodiment of technology
}

The increasing integration of wearable technologies with the human body raises neural and cognitive challenges and opportunities.

\section{Tamar R. Makin, Frederique de Vignemont and A. Aldo Faisal}

By exploiting robotics and information technology, teams of biomedical engineers are enhancing human sensory and motor abilities. Such augmentation technology - to be worn, implanted or ingested - aims to both restore and improve existing human capabilities (such as faster running, via exoskeletons), and to add new ones (for example, a 'radar sense'). The development of augmentation technology is driven by rapid advances in human-machine interfaces, energy storage and mobile computing. Although engineers are embracing body augmentation from a technical perspective, little attention has been devoted to how the human brain might support such technological innovation. In this Comment, we highlight expected neurocognitive bottlenecks imposed by brain plasticity, adaptation and learning that could impact the design and performance of sensory and motor augmentation technology. We call for further consideration of how human-machine integration can be best achieved.

Although there may be several ways to improve the effectiveness of augmentation technology, here we focus on embodiment - that is, the ability to process information through external objects at the sensory, motor and/or affective levels in the same way as the properties of one's own body parts ${ }^{1}$. Improving the embodiment of devices could potentially promote intuitive control, as well as facilitate learning, user satisfaction and long-term adoption of new technology. Embodiment may also reduce the risk of rejection of newer devices and facilitate the acquisition of knowledge in cognitive and motor control. Yet embodiment also opens up a plethora of theoretical and practical questions: From a neurocognitive perspective, will it be possible to successfully merge technology with the human body; that is, can we modulate sufficiently our body representations? What will embodied technology feel like to the user? What are the likely side effects (in particular, costs) of repurposing body representation to control augmentative technology? Can these side effects be minimized? Because augmentation technologies are still emerging, it is not surprising that such questions have largely been unexplored. Here, we argue that the next generation of augmentation technology needs to integrate understanding and methods from the cognitive sciences. Such a route would be a logical continuation of biomedical engineering efforts (such as those in radiological imaging or deep-brain stimulation) that have required a deeper understanding of biological constraints in order to achieve progress. In what follows, we rationalize why, in addition to typical biomechanical and neurophysiological knowledge, the design cycle of technologies for the enhancement of the human body to tackle disease and improve health ought to include neurocognitive considerations.

To modulate human perception and action, augmentation technology needs to interface with both the musculoskeletal system and the peripheral and central nervous systems. For example, to control artificial limbs, one can use a separate body part to mechanically control the artificial limb (Fig. 1a,b), peripheral signal processing of the residual limb movement (Fig. 1c), or direct motor-cortical readouts (Fig. 1d). Originally, artificial arms were designed to imitate the function of a tool (such as a mechanically powered 'hook', controlled by shifting the contralateral shoulder; Fig. 1b). Technological advancement in electromyography and signal processing has enabled control of hand-like prosthetics via residual muscles (Fig. 1c). However, limitations in the readout of electromyography signals result in non-intuitive and coarse control (for instance, users may be required to switch between discrete hand grasps). Current developments aim to enable the user to control multiple joints of a bionic hand simultaneously and to do it smoothly (that is, through proportional control of the joints) ${ }^{2}$. Still, the use remains counterintuitive at first and requires training.

Bionic prosthetics are a prime model for exploring neurocognitive considerations, and highlight the complexities of learning how to operate augmentative technology in real-world settings. Surprisingly, despite great progress from a technological standpoint in the design of prosthetics, and the fact that amputees are under societal and physical pressure to wear their prostheses, only $45 \%$ of all arm amputees choose to use their prosthesis throughout the day ${ }^{3}$. Of these, many prefer the simpler, low-tech devices (only $20 \%$ take advantage of technologically advanced robotic prosthetics $\left.{ }^{3}\right)$. The causes for the rejection of prostheses are manifold, and include awkward control over the device, lack of tactile feedback, and long, complex training requirements. Both clinicians and engineers have highlighted the possibility that amputees reject their prostheses because they do not feel like a real body part. The implicit (yet untested) assumption is that if prostheses were perceived by the user or represented by their brain as parts of their body, this should enhance ease of learning, control, usage and satisfaction ${ }^{4}$. Artificial limbs are therefore an ideal test-bed for exploring embodiment of wearable technology (Fig. 2). Although artificial limbs were originally designed to substitute a missing limb, today's wearable supernumerary robotic fingers and arms (Fig. 2a) are designed to augment the able human body 5,6 . Yet controlling robotic limbs independently from the natural ones remains a challenge.

We believe that technological embodiment could be a powerful driver for technological improvement, allowing engineers to repurpose the resources already available for operating our body and senses and thereby mitigating challenges in interfacing and training. However, at present, embodied technology remains a concept that needs to be experimentally explored. In the following paragraphs, we highlight key challenges in perceptual and motor embodiment at three distinct 
yet interdependent levels (although depending on specific technological implementations, some levels may be more important than others).

Phenomenological embodiment. Is it necessary, or even possible, to experience a technological device - such as an artificial arm - as a part of one's body? Does it entail that one should be able to feel pleasure and pain through it, or at least experience sensations that motivate subsequent behaviours, such as a reflexive withdrawal movement? Although phenomenological embodiment is likely to increase the acceptance of augmentation technology, it also raises potential confounds. First, the experience of the body as our own is relatively rigid7; contrary to folk wisdom, we do not experience ownership over most of the tools that we use ${ }^{8}$ (even those with which we are proficient with), suggesting that phenomenological embodiment may be difficult to achieve. Second, embodiment could also be limiting; for instance, the range of actions carried out with augmentation technology would be far more limited if we were to protect it in the way we protect the human body. And third, conscious embodiment might not be advantageous for all augmentation technology; for example, a perfect cochlear implant that restores hearing should remain phenomenologically transparent to the user.

Cognitive embodiment. Information about the human body arrives from multiple senses that merge onto a unified and consistent representation (sometimes called body schema or body image). The embodiment of augmentation technology will thus require integration of device inputs and outputs at the multisensory level as an integrated part of the body representation. Augmentation technology might also allow us to perceive information that is physiologically not available (such as ultraviolet wavelength), and to carry tasks that we are unable to perform (for example, tri-manual coordination using a supernumerary artificial arm; Fig. 2a). By piggybacking on the integrative mechanisms of body representation, augmentation-technology devices such as artificial arms will be able to achieve more complex and dynamic representations, encompassing both native and artificial abilities. However, there may be innate body-representation constraints that might preclude the effective adjustment to additional senses or limbs.

Human cognitive capacity - in particular, attention - is inherently constrained ${ }^{9}$. Whereas cognitive load is a design consideration in some areas of rehabilitation engineering, the neurocognitive mechanisms that underlie cognitive capacity and filtering mechanisms are seldom explored. This is important when augmenting perception, as data deluge from artificial sensory sources could overload the human brain. One potential solution involves sensory adaptation - the removal of the predictable components of a signal in order to enhance error signals - a key feature of the brain's inherent filtering mechanisms for making predictions ${ }^{10}$. Analogously, the brain's motor system exploits the ability to make predictions to account for time delays between perception and action or to compensate for noise in them. Another way to cope with data deluge involves active sensing. For example, visual attention is paired with the ability to actively control the eye's focus on high-resolution sensory data (through the eye's fovea, responsible for sharp vision). Such redundancy-reduction mechanisms are evolutionary design principles of organisation in the human sensory and motor pathways, and would likely have to be mirrored in novel artificial senses such as ultrasonic hearing or radar vision, or in substitutionary sensory (Fig. 2b) and motor devices (Fig. 2c,d). Yet piggybacking on the brain's filtering mechanisms implies strong design requirements. For instance, the filtering mechanism of sensory cancellation can help to filter out the tactile perceptions caused by the body's own movements from external effects. If such filtering methods are transparently and neuromorphically implemented, augmented senses are more likely to be felt as if they were embodied. This would require the low-latency control of artificial limb movements $(<70 \mathrm{~ms}$ time delay), placing severe constraints on many, but not all, of the current interfacing technologies. Indeed, latencies for eye-tracking-based intention decoding are under $10 \mathrm{~ms}$, which has enabled the high-precision control of robotic actuators in 3D with short training times ${ }^{11}$ (Fig. 2e).

Neural embodiment. The embodiment of augmentation technology could take advantage of neural resources devoted to controlling the human body. For technology that restores function (as with prostheses; Fig. 1d), the user might benefit from unused neural resources of the nervous system (such as the motor cortex) to control the missing limb. This opportunity would offer a natural substrate for the control of augmentation technology ${ }^{12}$, thereby also facilitating motor control and learning. The benefits of embodiment are, however, less apparent when considering technology that augments either the senses or actuation modalities (for example, supernumerary limbs, Fig. 2a). By hijacking the neural resources that control body parts, augmentation technology could even disorganize existing neural representations, causing potential damage to the control of such body parts (maladaptive plasticity ${ }^{13,14}$ ). An alternative to embodiment is harnessing the physiological processes of learning (brain plasticity) to use more general — or even arbitrary — neural interfaces; for instance, after extensive training, the visual cortex can be hijacked to control an artificial limb ${ }^{15}$.

Although embodiment is beneficial — and perhaps even necessary — for the long-term adoption of augmentation technology, by itself it will not be sufficient. The embodiment of technology cannot substitute practical considerations, such as setup-time, calibration, low latency and speed of movement. For example, the first bionic Olympics games - the Cybathlon 2016 — pitted for the first time competing technologies against each other in the same benchmark activities, inspired by daily-life tasks. Strikingly, the winning prosthetic arm was not bionic, but instead powered by mechanical substitution using movements from the arm and torso (as the prosthetic in Fig. 1b). Although simple in its features, this mechanical substitution interface had no latency, and offered fine-grained proportional control, unsurpassed by cuttingedge bionic technology. Similarly, many exoskeleton users (Fig. 2c) prefer to use their wheelchair rather than a lowerlimb exoskeleton designed for superior leg force (typically at the expense of speed).

The embodiment of augmentation technology can help us to harness the ability of the nervous system to learn and adapt to new circumstances (adaptive plasticity ${ }^{13}$ ), substantially boosting the implementation of the technology. And successful implementation could profoundly change the way we interact with the environment, and lead to significant healthcare and societal impact. But the realization of this vision could be restricted by the ability of the human brain to successfully and safely control augmentation technology. Poor neurocognitive design could lead to side effects as a result of limitations in the brain's capacity to operate the body and senses (maladaptive plasticity), which could lead to serious and long-lasting effects $^{14}$. Hence, it is important to consider the effects of embodied technology under dynamic circumstances. For 
instance, would a factory worker wearing supernumerary robotic arms throughout their shift be able to properly re-adapt their body movements when driving home afterwards? Future research should also consider potentially vulnerable populations from a neurocognitive perspective. For example, how will the vastly more plastic brain of children and adolescents be impacted by exposure to embodied technology?

Ultimately, the success of augmentation technology will be judged by user uptake and by the improvements in their quality of life. A systematic identification of the main benefits and boundaries of embodiment from a multidisciplinary perspective will help to improve the next generations of augmentation technology.

Tamar R. Makin ${ }^{1}$, Frederique de Vignemont ${ }^{2}$ \& A. Aldo Faisal ${ }^{3}$ are at the ${ }^{1}$ FMRIB Centre, Nuffield Department of Clinical Neurosciences, University of Oxford, Oxford, UK, and Institute of Cognitive Neuroscience, University College London, London, UK, ${ }^{2}$ Institut Jean Nicod (CNRS - ENS - EHESS), Institut des Etudes Cognitives, PSL,, Paris, France, and ${ }^{3}$ Departments of Bioengineering and of Computing, Imperial College London, London, UK.

E-mail: tamar.makin@ndcn.ox.ac.uk

\section{References}

1. de Vignemont, F. Conscious Cogn. 20, 82-93 (2011).

2. Xiloyannis, M., Gavriel, C., Thomik, A. A. C, \& Faisal, A. A. Gaussian Process Autoregression for Simultaneous Proportional MultiModal Prosthetic Control with Natural Hand Kinematics, IEEE Transactions on Neural Systems \& Rehabilitation Engineering (2017).

3. Jang, C. H. et al. Ann. Rehabil. Med. 35, 907-921 (2011).

4. Tyler, D. J. Curr. Opin. Neurol. 28, 574-581 (2015).

5. Wu, F. \& Asada, H. Supernumerary Robotic Fingers: An Alternative Upper-Limb Prosthesis. Proceedings of the ASME Dynamic Systems and Control Conference (2014). doi:10.1115/DSCC2014-6017

6. Llorens-Bonilla, B. \& Asada, H. Control and Coordination of Supernumerary Robotic Limbs Based on Human Motion Detection and Task Petri Net Model. Proceedings of the ASME Dynamic Systems and Control Conference (2013). doi:10.1115/DSCC2013-4083

7. Kikkert, S. et al. eLife 5, e15292 (2016).

8. Holmes, N. P. Exp. Brain Res. 218, 273-282 (2012).

9. Wolfe, J. M. \& Horowitz, T. S. Nat. Rev. Neurosci. 5, 495-501 (2004).

10. Faisal, A. A., Selen, L. P. J. \& Wolpert, D. M. Nat. Rev. Neurosci. 9, 292-303 (2008).

11. Abbott, W. W. \& Faisal, A. A. J. Neural Eng. 9, 046016 (2012).

12. Bensmaia, S. J. \& Miller, L. E. Nat. Rev. Neurosci. 15, 313-325 (2014).

13. Nava, E. \& Röder, B. Prog. Brain Res. 191, 177-194 (2011).

14. Münte, T. F., Altenmüller, E. \& Jäncke, L. Nat. Rev. Neurosci. 3, 473-478 (2002).

15. Neely, R. Koralek, A. C., Costa, R. M. \& Carmena. J. M. Operant control of primary visual cortex activity using a neuroprosthetic task in rodents. Neuroscience 2015, Society for Neuroscience, Meeting abstract 111.12 (2015).

Competing interests

The authors declare no competing financial interests.

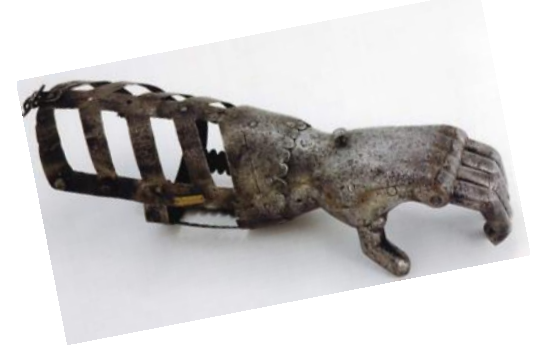

Mechanical prosthesis

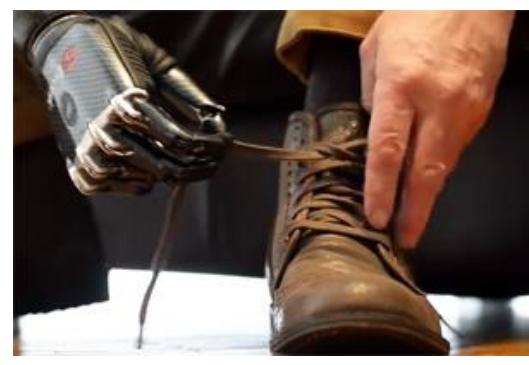

Myoelectric prosthesis

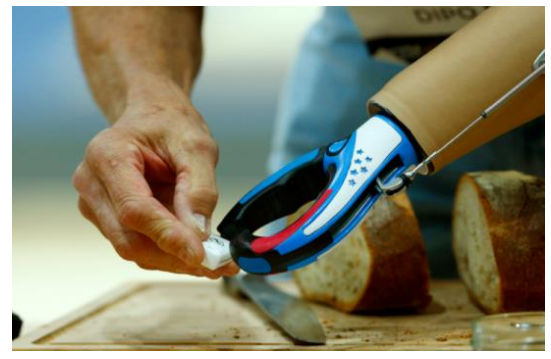

Body-powered prosthesis

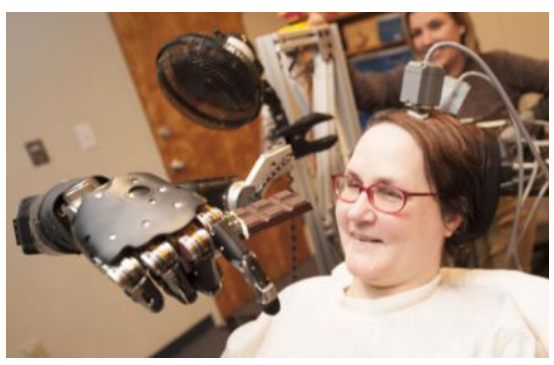

Brain-implant-controlled prosthesis

Figure 1 | Artificial arms for restoring hand function. a, An early mechanical prosthesis (ca. 1580) with movable joints for changing the static hand pose. $\mathbf{b}$, A prosthetic arm that uses motions of the contralateral arm to operate the motion of the hook-like gripper. $\mathbf{c}, \mathbf{A}$ bionic prosthetic arm that uses the readout of surface electromyography of the residual muscle to control pre-defined hand motions. $\mathbf{d}$, Control of a multi-degree-of-freedom robotic arm by means of microelectrode array implants in the neocortex. Images courtesy of a, Peter Finer; b, TRS Inc.; c, Bebionic; d, University of Pittsburgh Medical Center. 
[Note to art editor: panels $a$ and $b$ are those on top row; $c$ and $d$ are those in the bottom row. High-resolution images are in: a, http://myarmoury.com/talk/viewtopic.php?t=7161; b, http://www.trsprosthetics.com/cybathlon-winnerbob-radocy; c, http://www.geek.com/wp-content/uploads/2013/03/thandtshoe.jpg; d,

http://www,nature.com/news/us-regulators-move-on-thought-controlled-prosthetics-1.16407]

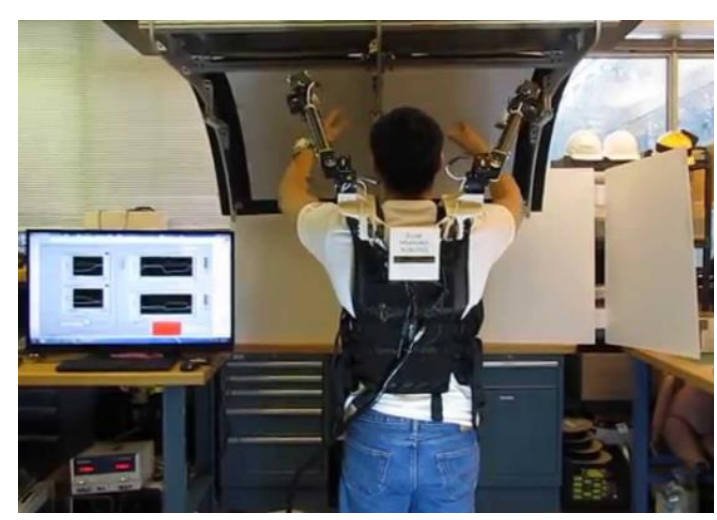

Supernumerary robotic arms

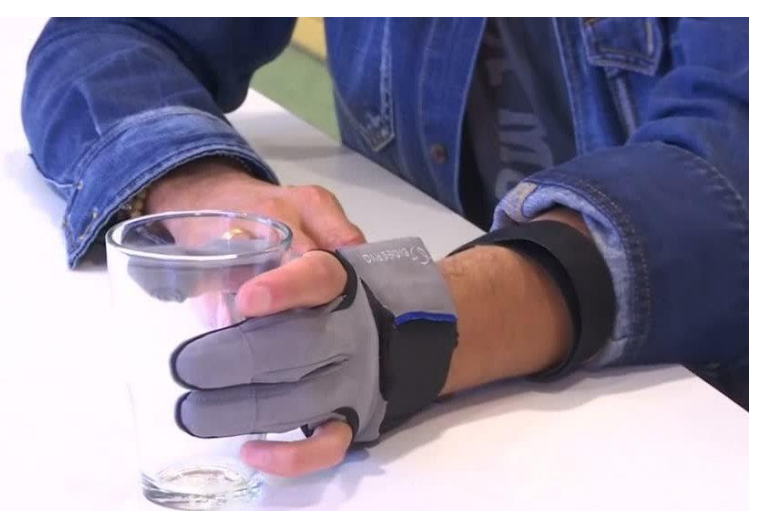

Soft-robotics hand orthotics
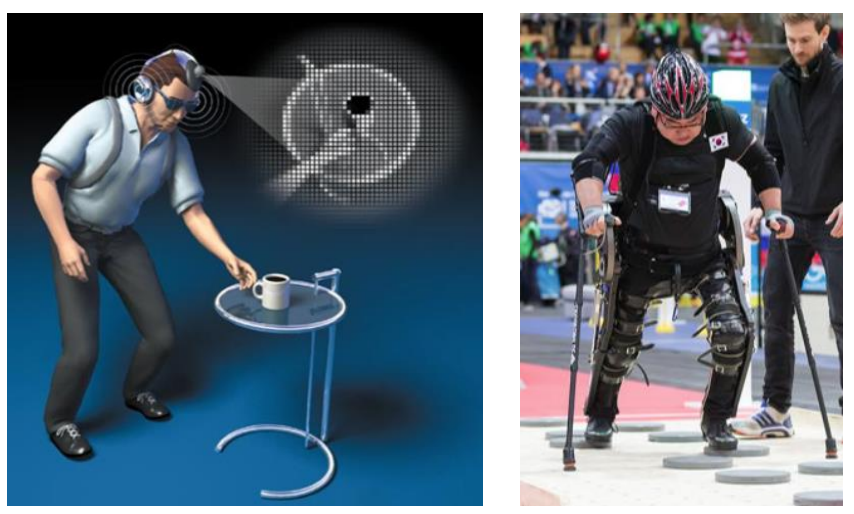

Sensory substitution

Lower-limb exoskeleton

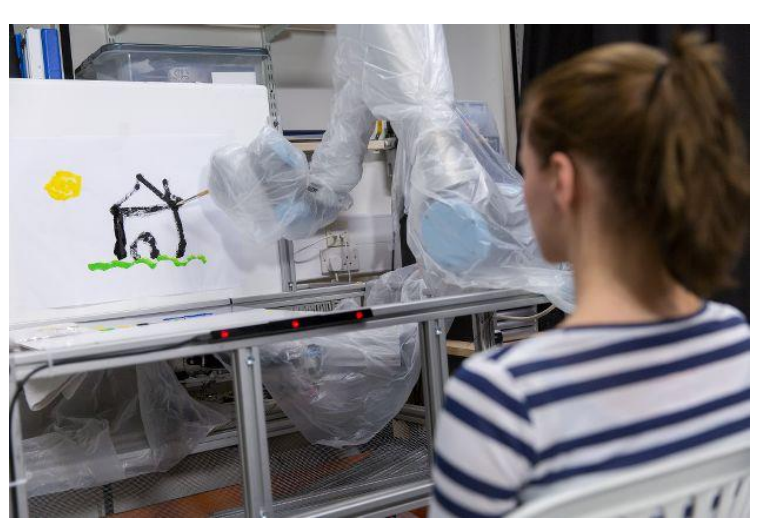

Gaze-controlled robotic arm

Figure 2 | Human augmentation in perception and action. a, A supernumerary robotic arm mounted on the back of a worker assists it in difficult assembly situations. b, Sonification of camera images can make the blind see a scene. c, Lower-limb exoskeleton enables a paraplegic man to walk through an obstacle course at the Cybathlon bionic Olympics. d, Soft-robotic orthotic glove enables paralyzed stroke patients to enhance a hand's grip strength whenever the hand is grasping an object. e, The decoding of action-intention from 3D eye-tracking analysis enables multi-degree-of-freedom control of a robotic arm. Images courtesy of a, d'Arbeloff laboratory (MIT); $\mathbf{b}$, Peter B. L. Meijer; c, ETH Zurich/A. Della Bella; d, BioServo Ltd.

[Note to art editor: Panels a, $b$ and $c$ are those on the top row. High-resolution images are in a, https://i.ytimg.com/vi/klkGDKKRPYU/maxresdefault.jpg (we may be able to obtain a higher-resolution version when seeking permission); b, https://www.seeingwithsound.com/sensub.htm c, http://www.cybathlon.ethz.ch/en/for-themedia/photo-gallery.html; d, http://lifeboat.com/blog.images/the-bioservo-or-soft-extra-muscles-sem-glove-mimicsthe-human-hand.jpg. Panel $\mathrm{e}$ is an original photo from one of the authors.] 